less than that corresponding to entry at Manchester.

It must be noted that the increasing atmospheric absorption at low neutron energies probably affects the neutrons produced at Manchester by the great solar flare. The production of neutrons at the top of the atmosphere may then be greater than that estimated in $\S 3$.

\section{Conclusion}

We conclude that the study of neutron intensity variations at sea level is a useful method for detecting changes in the energy of cosmic rays incident on the earth, and in particular in the lower end of the energy spectrum, down to rather less than $1000 \mathrm{MeV}$. We are now building a permanent neutron recorder to be operated continuously at Manchester.

We are very glad to present this work in homage to Professor Regener who has inspired and conducted so much work on the cosmic rays in the earth's atmosphere. In doing so, we voice the congratulations of Professor P.M.S. B lackett and the Manchester school of cosmic ray physics.

\title{
Vergleich von Reichweite und Energiespektrum der durchdringenden Teilchen der Ultrastrahlung
}

\author{
Von H. D. RathgebeR* \\ Research Fellow, University of Melbourne; Australia \\ (Z. Naturforschg. 6 a, 598-602 [1951]; eingegangen am 10. Juli 1951) \\ Erich Regener zum 70. Geburtstage
}

\begin{abstract}
Die Absorption des durchdringenden Anteils der Ultrastrahlung in Wasser wird mit der Ablenkung der geladenen Ultrastrahlungsteilchen im Magnetfeld verglichen. Es wird gezeigt, daß keine plötzliche Änderung des Energieverlustes von $2 \cdot 10^{8}$ bis über $5 \cdot 10^{10} \mathrm{eV}$ eintritt, und es wird daraus geschlossen, daß der größte Teil der Strahlung in diesem Bereich aus $\mu$-Mesonen besteht. Die Ergebnisse sind weder mit der Bethe-Bloch-Theorie der spezifischen Ionisation noch mit der die Polarisation berücksichtigenden von Fermi zu vereinbaren. Sie werden am besten durch einen spezifischen Energieverlust gedeutet, der sich von $5 \cdot 10^{8}$ bis $3 \cdot 10^{10} \mathrm{eV}$ nicht ändert und $(2,0 \pm 0,1) \cdot 10^{6} \mathrm{eV} \mathrm{g}^{-1} \mathrm{~cm}^{2}$ beträgt. Unmittelbare Messungen der wahrscheinlichen spezifischen Ionisation mit Proportionalzählrohr stimmen damit überein.
\end{abstract}

I m Jahre. 1929 veröffentlichte Re g e n e ${ }^{1}$ die Ergebnisse seiner Absorptionsmessungen im Bodensee. Die stetige Abnahme der Ionisation bis zu Tiefen von $250 \mathrm{~m}$ zeigte zum ersten Male eindeutig das große Durchdringungsvermögen der Ultrastrahlung, das dasjenige aller bekannten Strahlen übersteigt. Da die $\gamma$-Strahlen die zur Zeit größte bekannte Durchdringungsfähigkeit besaßen und ihr Absorptionskoeffizient mit der Wellenlänge abnimmt, lag es nahe anzunehmen, daß die Ultrastrahlung eine Wellenstrahlung von noch kürzerer Wellenlänge sei. Die Zählrohrkoinzidenzmessungen von $\mathrm{B}$ othe und Kolhörs te ${ }^{2}$ und die Wilsonkammeraufnahmen von $\mathrm{Sko-}$ belzyn ${ }^{3}$ waren die ersten einer langen Reihe von Experimenten, die bewiesen, daß die Ultrastrahlung

1 E. R e g e n e r, Naturwiss. 17, 183 [1929].

2 W. B othe u. W. Kolhörster, Naturwiss. 16, 1045 [1928].

3 D. Z. S k obelzy n, Z. Physik 43, 354 [1927]. in Meereshöhe hauptsächlich aus geladenen Teilchen besteht. Die experimentelle Bestätigung der Quantenelektrodynamik durch die Beobachtung der Elektronenschauer zeigte, daß die Energieverluste von elektromagnetischer Strahlung und leichten geladenen Teilchen außerordentlich rasch mit der Energie zunehmen. Diese Theorie sagt weiter aus, daß nur der Energieverlust von ungeladenen oder schwerer geladenen Teilchen so klein sei, daß sie mehrere hundert Meter Wasser zu durchdringen vermögen. Die Absorptionskurve in Wasser fand ihre Deutung als Reichweitekurve solcher Teilchen, den $\mu$-Mesonen, von denen wir wissen, daß sie zerfallen. Es entsteht nun die Frage nach der Natur des kleinen Bruchteils der Strahlung, die in große Tiefen eindringt.

Eine Eigenschaft, die solche Aussagen zuläßt und die experimentell bestimmt werden kann, ist die Beziehung zwischen Reichweite und Energie der Teilchen. Die gleichzeitige Messung an ein und dem- 
selben Teilchen von Energie und Reichweite ist nur bis zu Reichweiten von der Größenordnung von einem Meter praktisch möglich. Selbst mit einem Bleiabsorber ist also nur ein kleiner Bruchteil der größten Reichweite experimentell zugänglich. Im Jahre 1935 haben zuerst $\mathrm{S}$ tre e $\mathrm{t}, \mathrm{W}$ o o d w a rd und Stevens on ${ }^{4}$ gezeigt, wie die Reichweite-Energie-Beziehung durch Vergleich der Absorptionskurve mit dem integralen Energiespektrum gewonnen werden kann. Mit Hilfe seines Energiespektrums hat später B la cke t $t^{5}$ diese Methode weiter ausgebaut. Seitherige Fortschritte sowohl in der Messung der Absorptionskurve als auch des Energiespektrums erlauben jetzt, die Genauigkeit dieser Ergebnisse zu erhöhen und sie bis zu höheren Energien auszudehnen.

\section{Methode}

Die Absorptionskurve der Ultrastrahlung in einem Medium gibt die·Intensität der Teilchen an, deren Reichweite größer als die Meßtiefe ist. Andererseits kennen wir aus dem integralen Energiespektrum die Intensität der Teilchen, deren Energie einen bestimmten Wert überschreitet. Durch Vergleich der Reichweite mit der Energie, bei gleichen Intensitäten, können wir die Reichweite-Energie-Beziehung erhalten.

Wir wollen nun die Bedingungen erörtern, unter denen dieses Verfahren gilt, und uns dabei im wesentlichen an Blacketts ${ }^{5}$ Analyse anschließen. In jedem Reichweitenbereich soll nur eine Teilchenart so vorherrschend sein, daß der Einfluß der anderen vernachlässigbar ist. Außerdem soll in jedem solchen Bereich die Reichweite eine eindeutige gleichmäßig ansteigende Funktion der Energie sein. Zur Erfüllung dieser Bedingungen ist es notwendig, daß der Energieverlust im allgemeinen nur in kleinen Bruchteilen der Gesamtenergie erfolgt.

Wir werden zuerst aus den bekannten Messungen die Absorptionskurve für den harten Anteil der Ultrastrahlung ableiten. Wir definieren diesen, wie gebräuchlich, als denjenigen Anteil, der $10 \mathrm{~cm}$ Blei durchdringt, d. h. der größte Teil der Elektronkomponente wird ausgeschlossen. Das integrale Energiespektrum der Teilchen, die ebenfalls $10 \mathrm{~cm}$ Blei durchdringen, wird aus Ablenkungsmessungen im Magnetfeld ermittelt.

4 J. C. Street, R. H. W o odward u. E. C. Steve n s o n, Physic. Rev. 47, 891 [1935].

5 P. M. S. B l a ckett, Proc. Roy. Soc. [London], Ser. A 159, 19 [1937].

6 A. E hmert, Z. Physik 106, 751 [1937].
Die Reichweite-Energie-Beziehung wird dann durch graphischen Vergleich der beiden Kurven erhalten. Die Berechnung des Energieverlustes für die verschiedenen Energiebereiche folgt daraus.

Die Ergebnisse werden zum Schluß mit theoretischen Berechnungen des Energieverlustes und den Ergebnissen anderer experimenteller Methoden verglichen.

\section{Die Absorptionskurve}

In Weiterführung von $\mathrm{R}$ e g e $\mathrm{n}$ e r $\mathrm{s}$ Versuchen hat Ehmert ${ }^{6}$ die Absorption der Ultrastrahlung im Bodensee mit Zählrohrkoinzidenzen gemessen. Unter

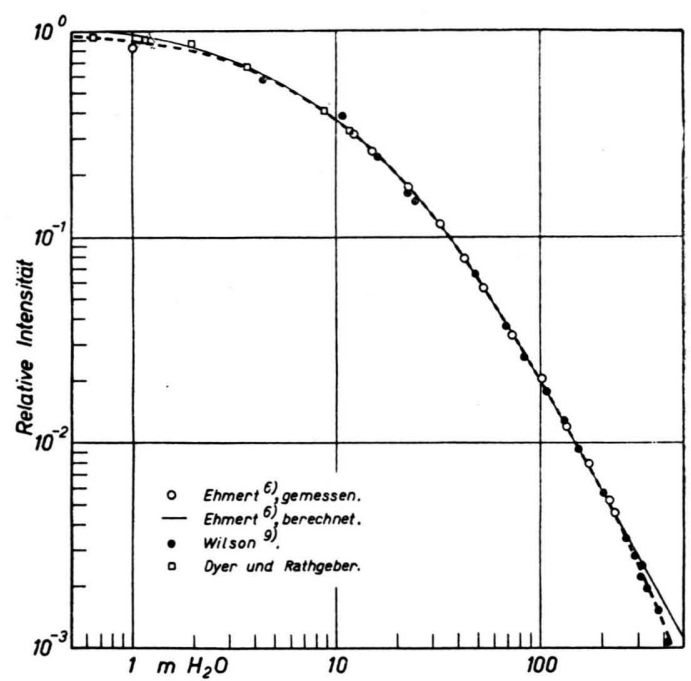

Abb. 1. Absorptionskurve für senkrechten Einfall der durchdringenden Komponente der Ultrastrahlung.

Berücksichtigung der Geometrie der Apparatur hat er daraus die Absorptionskurve für senkrechten Einfall berechnet.

Aus den folgenden Gründen haben wir diese Kurve zur Grundlage unserer Analyse genommen. Erstens ist sie in Material von niedriger Kernladung gemessen; die Strahlungsverluste, wenn überhaupt vorhanden, sind so klein als möglich. Zweitens ist die Masse des Absorbers genau bekannt, sowohl der Dichte als der Tiefe nach.

Die aus den Formeln für senkrechten Einfall berechnete Intensität ist in Abb. 1 als Funktion der Wassertiefe aufgetragen. Diese Absorptionskurve wurde ohne Absorber zwischen den Zählrohren gemessen; sie enthält also die Elektronenkomponente, die wir von dem Vergleich ausschließen wollen. Einige mit $5 \mathrm{~cm}$ Blei zwischen den Zählrohren gemessene Intensitäten zeigen, daß innerhalb der stati- 
stischen Fehler in allen Tiefen der gleiche Bruchteil der Strahlung absorbiert wird. Dies wird durch Messungen von $\mathrm{Clay}^{\prime}$ und $\mathrm{Gemert^{7 }}$ über dem in Frage kommenden Tiefenbereich bestätigt für $\mathrm{Ab}$ sorber von 5 und $10 \mathrm{~cm}$ Blei. Es ist weiter von Ros si und $\mathrm{Klap} \mathrm{man}^{8}$ berechnet worden, daß das Verhältnis von Sekundärelektronen zu den sie erzeugenden Mesonen nicht von der Energie der Mesonen abhängt. Wir sind also berechtigt, in Tiefen, wo Elektronen und Mesonen im Gleichgewicht sind, die durchdringende Ultrastrahlung als proportional zur Gesamtstrahlung anzunehmen. Für unseren Zweck, Vergleich mi dem Energiespektrum, genügt es, die relative Intensität zu kennen.

Es ist zu erwarten, daß bei dem Übergang von Luft zu Wasser die Elektronenkomponente nicht im Gleichgewicht mit der Mesonenkomponente steht. Dieser Übergang wird bis zu $11 \mathrm{~m}$ Wassertiefe erfaßt ${ }^{9}$. Die weiche Komponente wird durch $10 \mathrm{~cm}$ Blei zwischen den Zählrohren einer Koinzidenzapparatur von $10^{\circ}$ Öffnungswinkel absorbiert.

Für Tiefen über $240 \mathrm{~m}$ werden die Bodenseemessungen mit den Messungen von Wils o n ${ }^{10}$ in einem Bergwerk ergänzt. Bei dem Versuch, die Ehmertsche Kurve mit der von Wilson zur Deckung zu bringen, fanden wir, daß es notwendig war, die von Wilson berechnete Äquivalenttiefe mit 0,95 zu multiplizieren. Dies ist im Einklang mit den Berechnungen von $\mathrm{H}$ a $\mathrm{ll}$ und $\mathrm{H}$ a $\mathrm{l}$ p e r $\mathrm{n}^{11}$, die zeigen, daß der spezifische Energieverlust für zunehmendes Atomgewicht abnimmt.

Die obigen Erwägungen führen zur Konstruktion der gestrichelt gezeichneten Kurve, die als proportional zur durchdringenden Ultrastrahlung angenommen wird.

\section{Das Energiespektrum}

Es liegen aus den letzten Jahren mehrere Bestimmungen des Energiespektrums bis über $2 \cdot 10^{10} \mathrm{eV}$ vor: von $\mathrm{O}$ wen und $\mathrm{Wils}$ on ${ }^{12}$ und von $\mathrm{Caro}$, Parry und Rathgeber ${ }^{13}$ (im folgenden mit CPR abgekürzt), die mit Zählrohrapparaturen die Ablenkung der geladenen Ultrastrahlungsteilchen im Magnetfeld gemessen haben, und von Glaser,

7 J. Clay u. A. v. Ge mert, Physica 6, 497 [1939].

8 B. Rossi u. S. J. K lapman, Physic. Rev. 61, 414 [1942].

9 Noch unveröffentlichte Messungen gemeinsam mit A. J. Dy e r.

10 V. C. Wils o n, Physic. Rev. 53, 340 [1938].

11 O. Ha lpern u. H. H a ll, Physic. Rev. 73, 477 [1948].
$\mathrm{H}$ amermesh und $\mathrm{S}$ a f onovit, die dafür zwei Wilsonkammern benutzt haben.

Wir werden zum Zweck der vorliegenden Arbeit ausschließlich die CPR-Messungen benutzen, aus denen die vier integralen Energiespektren der Abb. 2 entnommen sind. Es ist klar ersichtlich, daß mit steigender Feldstärke, d. h. steigendem Auflösungsvermögen, die Spektren bei hohen Energien schneller und schneller abfallen. Da sie einer Grenzkurve zustreben, sind die Messungen von höchstem Auflösungsvermögen vorzuziehen. Deshalb haben wir die

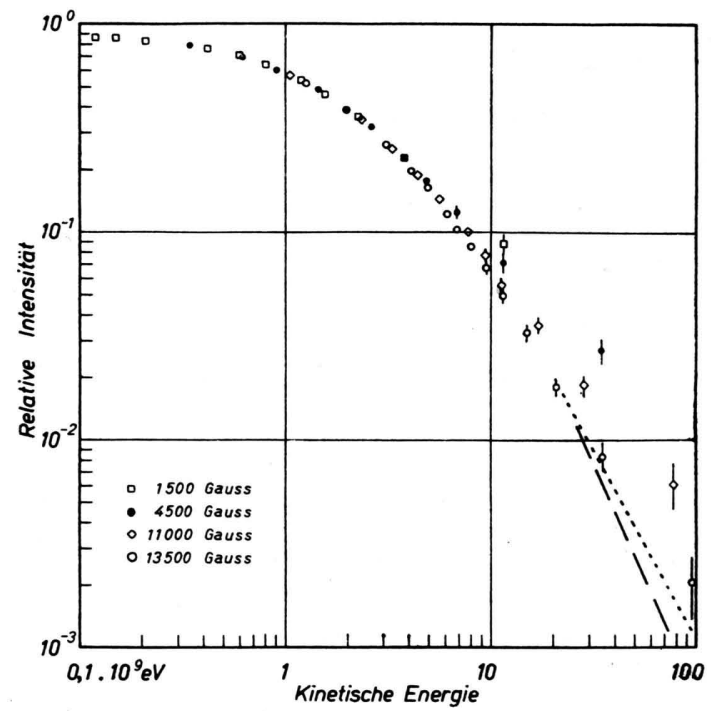

Abb. 2. Energiespektrum der durchdringenden Teilchen der Ultrastrahlung.

Messungen von $\mathrm{O}$ we $\mathrm{n}$ und $\mathrm{W} i \mathrm{ls}$ on, deren Auflösungsvermögen $\left(3 \cdot 10^{10} \mathrm{eV}\right)$ kleiner ist als das von CPR $\left(5 \cdot 10^{10} \mathrm{eV}\right)$, nicht benutzt. Ein weiterer Grund ist die unbekannte Korrektur für Teilchen kleiner Energie, die aus der Apparatur heraus abgelenkt worden sind. Wie die gute Überlappung der Energiespektren von CPR zeigt, ist diese Korektur hier richtig erfaßt worden.

In ihren Wilsonkammermessungen geben Glaser, $\mathrm{H}$ a mermesh und $\mathrm{S}$ af on ov ${ }^{14}$ ein Energiespektrum bis $10^{11} \mathrm{eV}$ an. Ein Vergleich ihres integralen Energiespektrums der Form $E^{-1,2}$ mit den CPRMessungen zeigt, daß es dem mit $11000 \mathrm{Gau}$ ge-

12 B. G. O w e n u. J. G. Wils o n, Proc. physic. Soc. Ser. A 62, 601 [1949].

13 D. E. Caro, J. K. P a r ry u. H. D. R a th ge ber, Nature [London] 165, 688 [1950]; Austral. J. Sci. Res. Ser. A 4, 16 [1951].

14 D. A. G las er, B. $\mathrm{H}$ a mermes h u. G. S a f onov, Physic. Rev. 80, 625 [1950]. 
messenen Spektrum der Form $E^{-1,3}$ entspricht. Dies, zusammen mit den Schwierigkeiten der Korrektur bei Energien unterhalb $10^{9} \mathrm{eV}$, rechtfertigt uns, diese Messungen ebenfalls als ungeeignet für unsere Zwecke auszuscheiden.

Die Gründe für die Unterschiede zwischen den mit verschiedenen Feldstärken gemessenen Spektren können leicht eingesehen werden. Nach den CPR-Mes-

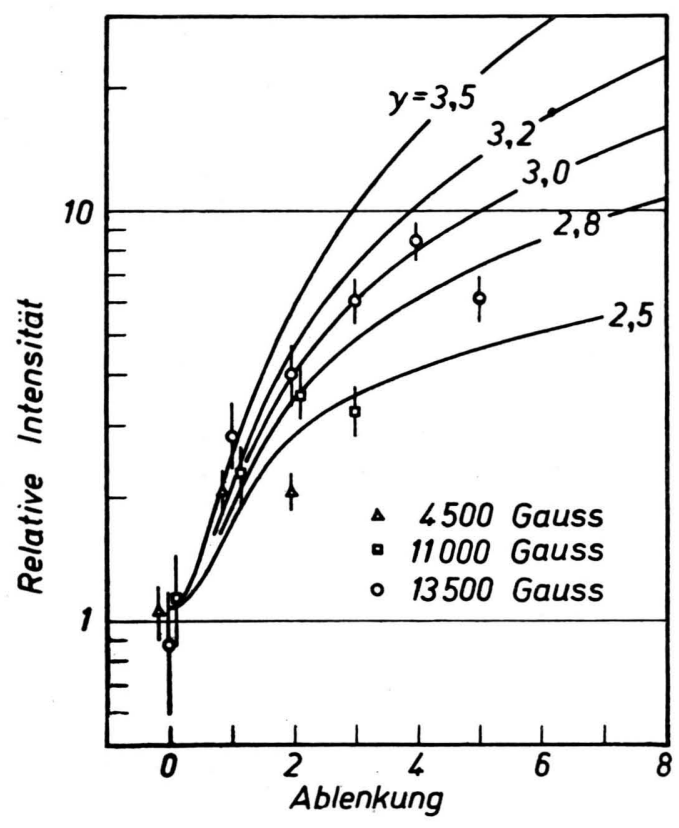

Abb. 3. Vergleich von Messung und berechneten Intensitäten als Funktion der Ablenkung.

sungen bei höchsten Feldstärken (s. ${ }^{13}$, Abb. 9) nimmt die Intensität für kleine Ablenkungen stark zu. Die Intensität an einem Meßpunkt kleiner Ablenkung besteht nun zuerst aus dem Beitrag von Teilchen der Nennenergie, d. h. derjenigen Energie, die der Ablenkung entspricht. Infolge Streuung und mangelndem Auflösungsvermögen werden aber auch Teilchen sowohl höherer als auch niedrigerer Energie zu der gemessenen Intensität am Meßpunkt beitragen. Es ist nicht so, daß die Teilchen höherer Energie ebensoviel dazu beitragen wie die niedrigerer; infolge der Intensitätszunahme überwiegt vielmehr der Beitrag der Teilchen niedrigerer Energie denjenigen der Teilchen höherer Energie und übersteigt sogar denjenigen der Nennenergie. Die mittlere Energie der bei einer bestimmten Ablenkung registrierten Teilchen ist deshalb nicht die Nennenergie, sondern eine kleinere. Dieser Unterschied zwischen Nennenergie und mittlerer Energie nimmt mit steigender Energie stark zu und führt zu einer langsameren Abnahme, als der Wirklichkeit entspricht.

Wir haben diese Schwierigkeiten in der folgenden Weise vermieden. Es wird ein differentielles Energiespektrum von auf die Apparatur einfallenden Teilchen von der Form $E^{-\gamma}$ angenommen. Daraus wird unter Berücksichtigung der Verteilung ohne Magnetfeld die Intensität als Funktion der Ablenkung numerisch berechnet. Diese Intensitätsfunktion wird über die Breite der Zählintervalle (bei CPR „counting units“) integriert. Durch dieses Verfahren wird das ursprüngliche Energiespektrum mathematisch derselben Verformung unterworfen wie in der Apparatur. Aus dem Vergleich so gewonnener Kurven (Abb. 3) mit den Meßergebnissen ist ersichtlich, daß $\gamma$ zwischen 2,8 und 3,2 liegt für die Punkte kleinster Ablenkung. Diese Grenzen, die bis $10^{11} \mathrm{eV}$ gültig sind, sind in Abb. 2 als punktierte und gestrichelte Geraden der Steilheit $2,8-1=1,8$ und $3,2-1=2,2$ für das integrale Spektrum gezeichnet.

\section{Die Reichweite-Energie-Beziehung und der Energieverlust}

In der Absorptionskurve und dem Energiespektrum besitzen wir die für unseren Vergleich notwendigen Unterlagen. Für diesen bestehen zwei Möglichkeiten: es kann entweder ein Punktpaar, für das die Reichweite und die Energie bekannt sind, zur Deckung gebracht oder eine möglichst gute Deckung beider Kurven angestrebt werden.

Für die erste Methode sind keine zuverlässigen Messungen für größere Absorberdicken vorhanden. Das Punktpaar für keinen Absorber und verschwindende Energie leidet daran, daß die Intensität der Teilchen von einer Energie $<5 \cdot 10^{8} \mathrm{eV}$ in den CPRMessungen infolge Streuung zu klein ist.

Wir haben die zweite Methode in Abb. 4 angewandt. Die auf durchsichtiges Logarithmenpapier gezeichneten Abb. 1 und 2 wurden so lange parallel verschoben, bis eine zufriedenstellende Deckung erreicht wurde. Wie ersichtlich, liegen die Unterschiede zwischen 5 und $10 \%$ in der Intensität bei niedrigen Energien in der erwarteten Richtung. Es darf dabei auch nicht vergessen werden, daß für $\mu$-Mesonen von Energien $<2 \cdot 10^{8} \mathrm{eV}$ die Energieverluste stark ansteigen. Es läßt sich abschätzen, daß der Einfluß dieses Effektes auf die Proportionalität von Reichweite und Energie, die durch die Überdeckung angenommen wird, bei ungefähr $5 \cdot 10^{8} \mathrm{eV}$ verschwindet.

Wie ersichtlich, decken sich die Kurven innerhalb der Meßfehler über den ganzen weiteren Verlauf bis 
zu $10^{11} \mathrm{eV}$. Erstens haben wir aus der Stetigkeit den Schluß zu ziehen, daß wir es mit einer in bezug auf die Energieverluste homogenen Strahlung zu tun haben. Denn es ist sehr unwahrscheinlich, daß Unterschiede in dem Energieverlust zweier Strahlenarten gerade durch die Änderung ihrer relativen Anzahl kompensiert werden. Es wird daher der Hauptteil der Strahlung in dem untersuchten Bereich wie in Meereshöhe aus $\mu$-Mesonen bestehen.

Zweitens tritt im Unterschied zu B lacketts Ergebnissen $^{5}$ keine Zunahme des Energieverlustes

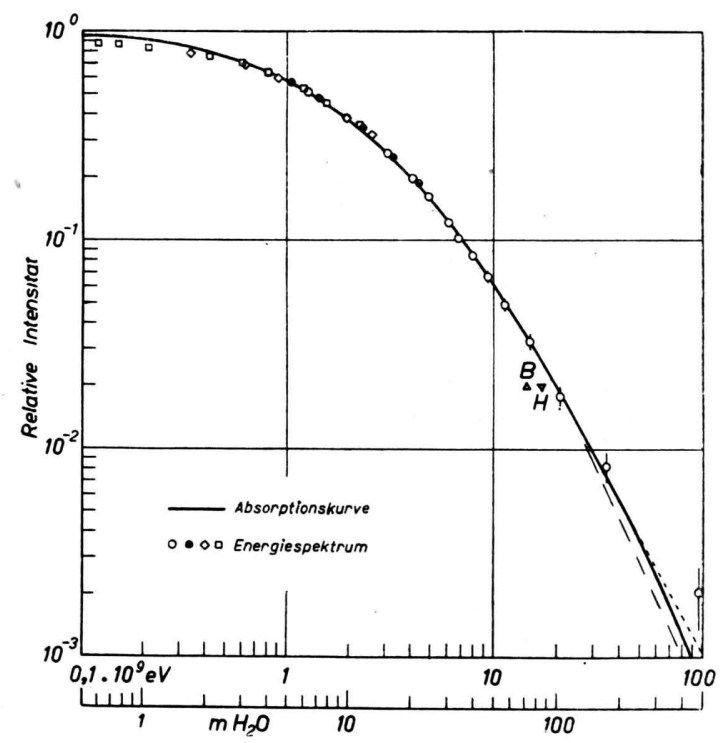

Abb. 4. Vergleich von Absorptionskurve und Energiespektrum.

mit zunehmender Energie bis zu $5 \cdot 10^{10} \mathrm{eV}$ auf, darüber hinaus ist keine Aussage infolge der Meßfehler möglich. Der Einsatz der Energieverluste durch Bremsstrahlung ist für $\mu$-Mesonen $\mathrm{zu} 10^{6}(\mu / \mathrm{m})^{2}$ $=4,5 \cdot 10^{10} \mathrm{eV}$ abgeschätzt worden. Unsere Ergebnisse zeigen, daß unterhalb dieser Grenze keine zusätzlichen Energieverluste einsetzen.

Nach der Theorie der Energieverluste durch unelastische Zusammenstöße ist zu erwarten, daß die Verluste durch Ionisation und Anregung mit steigender Energie langsam zunehmen. Wir wollen untersuchen, ob die Fehlergrenzen klein genug sind, um diese Zunahme nachzuweisen.

Wir nehmen an, daß der Energieverlust im Bereich von 0,5 bis $2 \cdot 10^{9} \mathrm{eV}$ mit einer mittleren Energie von
$1,25 \cdot 10^{9} \mathrm{eV}$ richtig sei. Aus der Bethe-Bloch-Theorie entnehmen wir, daß der Energieverlust von 1,25 bis $11 \cdot 10^{9} \mathrm{eV}$ der mittleren Energie des Bereiches von 2 bis $20 \cdot 10^{9} \mathrm{eV}$ um $29 \%$ zunimmt ${ }^{11}$. Die Zunahme der Reichweite von 2 bis $20 \cdot 10^{9} \mathrm{eV}$ wäre also um ebensoviel verkürzt, d. h. die Absorptionskurve sollte durch den Punkt B in Abb. 4 gehen. Die Energieverluste der $\mu$-Mesonen nehmen also langsamer $\mathrm{zu}$, als der Bethe-Bloch-Theorie entspricht.

Es ist von $\mathrm{Fermin} \mathrm{i}^{15}$ darauf hingewiesen worden, daß die Zunahme infolge Polarisation langsamer erfolgen sollte. Der Energieverlust ist von $\mathrm{H}$ a l per $\mathrm{n}$ und $\mathrm{H}$ a $\mathrm{ll}^{11}$ für verschiedene Medien berechnet worden. Da der Energieverlust in Wasser nach dieser Berechnung von 1,25 bis $11 \cdot 10^{9} \mathrm{eV}$ um $16 \%$ zunimmt, sollte die Absorptionskurve durch den Punkt $\mathrm{H}$ gehen. Selbst bei Berücksichtigung der Meßfehler und der Fehler bei der Anpassung der verschiedenen Energiespektren ist es bei weiterer Verschiebung der Kurven nicht möglich, die Absorptionskurve durch den Punkt $\mathrm{H}$ gehen $\mathrm{zu}$ lassen unter gleichzeitiger guter Anpassung bei Energien unterhalb $2 \cdot 10^{9} \mathrm{eV}$.

Durch gleichzeitige Messung der Ionisation von durchdringenden Teilchen mit einem Proportionalzähler und ihrer Ablenkung im Magnetfeld haben Goodman, Nicholson und Rathgeber ${ }^{16}$ festgestellt, daß die wahrscheinliche spezifische Ionisation langsamer zunimmt, als der Fermi-Theorie entspricht. Es sind hauptsächlich die entfernten Zusammenstöße, die meistens nur zu Anregungen führen, die in der Theorie zur relativistischen Zunahme des Energieverlustes beitragen. Es besteht somit die Möglichkeit, daß der Energieverlust trotz nahezu konstanter Ionisation zunimmt. Das Ergebnis unseres Vergleichs schließt diese Erklärung aus.

Das Verhältnis von Reichweite und Energie in Abb. 4 ergibt den spezifischen Energieverlust zwischen $5 \cdot 10^{8}$ und $3 \cdot 10 \mathrm{eV} \mathrm{zu}(2,0 \pm 0,1) \cdot 10^{6} \mathrm{eV} \mathrm{g}^{-1} \mathrm{~cm}^{2}$, während der theoretische Energieverlust in Wasser $2,05 \cdot 10^{6} \mathrm{eV} \mathrm{g}^{-1} \mathrm{~cm}^{2}$ am Minimum der Kurve beträgt. Diese gute Übereinstimmung schließt aus, daß $\mu$ Mesonen für einen Teil ihrer Reichweite ihre Ladung verlieren und so lange als neutrale Mesonen keine Energieverluste ${ }^{17}$ erleiden.

15 E. F e r m i, Physic. Rev. 57, 485 [1940].

16 P. Goodman, K. P. Nicholson u. H. D. Rathg e b e r, Proc. Physic. Soc. Ser. A 64, 96 [1951].

17 K. H. H ö c k e r, Ann. Physik 6, 353 [1949]. 